\title{
Importancia del Líquido Sinovial en la Articulación Temporomandibular y sus Implicancias en la Patología Articular
}

\author{
Value of Synovial Fluid in the Temporomandibular Joint \\ and its Implications in Articular Pathology
}

\author{
Veronica Iturriaga ${ }^{1,2,3}$; Paula Mena ${ }^{4}$; René Oliveros ${ }^{4}$ - Camila Cerda ${ }^{5}$ Daniela Torres $^{5} \&$ Mariano del Sol $^{6}$
}

ITURRIAGA, V.; MENA, P.; OLIVEROS, R.; CERDA, C.; TORRES, D. \& DEL SOL, M. Importancia del líquido sinovial en la articulación temporomandibular y sus implicancias en la patología articular. Int. J. Morphol., 36(1):297-302, 2018.

RESUMEN: El objetivo de este estudio fue realizar una revisión de la literatura respecto de la importancia del líquido sinovial en la articulación temporomandibular y sus implicancias en la patología articular. El líquido sinovial corresponde a un fluido viscoso y filante de color amarillento claro, compuesto principalmente por proteínas plasmáticas, componentes celulares, moléculas lubricantes, citoquinas, factores de crecimiento y enzimas. Es producido por la membrana sinovial y se encuentra en relación directa con las superficies articulares y la membrana sinovial, mediando las interacciones entre los tejidos de las articulaciones sinoviales. En la articulación temporomandibular desempeña un papel fundamental en el mantenimiento y regulación de la fisiología, a través de funciones lubricantes, reguladoras y metabólicas, disminuyendo el roce de las superficies articulares durante todos sus movimientos. En los trastornos temporomandibulares, la composición y función del líquido sinovial se puede ver alterada por cambios en los tejido de la articulación temporomandibular, estos cambios pueden resultar en una disminución en la capacidad de lubricar las superficies articulares y generar un ambiente catabólico en el interior de la articulación, contribuyendo conjuntamente al deterioro de éstas.

PALABRAS CLAVE: Líquido sinovial; Articulación temporomandibular; Trastornos temporomandibulares; Osteoartritis.

\section{INTRODUCCIÓN}

El líquido sinovial (LS) es un fluido viscoso transparente que constituye un ultrafiltrado del plasma. El LS contiene diversos componentes que le confieren propiedades lubricantes, metabólicas y regulatorias, que disminuyen la fricción y desgaste del cartílago articular (Hui et al., 2012), siendo característico de las articulaciones sinoviales. Las articulaciones sinoviales normalmente presentan un ligamento capsular (LC) junto a una membrana sinovial (MS) que contiene al LS en una cavidad que rodea al cartílago y hueso articular (Hui et al.).

Dentro de la articulaciones sinoviales se encuentra la articulación temporomandibular (ATM), donde el LS se comporta como una fina película de gel viscoso que lubrica la superficie articular (Kristensen et al., 2014). Se ha propuesto que la composición y función del LS se ven altera- dos en patologías articulares como la osteoartritis, produciendo un desequilibrio en la homeostasis de este.

El objetivo de este estudio fue realizar una revisión de la literatura respecto de la importancia del líquido sinovial en la articulación temporomandibular y sus implicancias en la patología articular.

Membrana y líquido sinoviales. La superficie interna de la cápsula de la ATM está tapizada por la MS de tejido conectivo laxo que produce y confina el LS, formándose en la etapa de maduración embriológica entre la 12 - 17 semanas de desarrollo (Wurgaft, 2003). La MS de la ATM tiene numerosas vellosidades y pliegues sinoviales que se proyectan a la cavidad articular. Hay que tener en consideración que las superficies óseas que forman la ATM no tienen

\footnotetext{
${ }^{1}$ Departamento de Odontología Integral Adulto, Universidad de La Frontera, Temuco, Chile.

${ }^{2}$ Programa Doctorado en Ciencias Médicas, Universidad de La Frontera, Temuco, Chile.

${ }^{3}$ Becario CONICYT, PFCHA/ Doctorado Nacional/2015-21151352.

${ }^{4}$ Estudiante Programa Especialidad en Trastornos Temporomandibulares y Dolor Orofacial, Universidad de La Frontera, Temuco, Chile.

${ }^{5}$ Especialidad en Trastornos Temporomandibulares y Dolor Orofacial, Universidad de La Frontera, Temuco, Chile.

${ }^{6}$ Centro de Estudios Morfológicos y Quirúrgicos (CEMyQ), Universidad de La Frontera, Temuco, Chile.

Esta investigación fue apoyada por CONICYT por medio de la beca Doctoral, PFCHA/2015-21151352.
} 
cartílago hialino, debido a la presencia de un disco de tejido conectivo fibroso denso, con fibras de colágenos tipo I el cual tiene entre sus funciones amortiguar las diversas fuerzas producidas en las diversos planos y direcciones durante la masticación, entre otras acciones.

La MS está ubicada en las zonas laterales de los compartimentos supra e infradiscal de la ATM, donde se almacena el LS que nutre las superficies articulares (Wurgaft). Está formada por dos capas: 1) La capa sinovial íntima, que limita con los espacios del LC y que contiene sinoviocitos tanto fagocíticos como secretorios; y 2) La capa sinovial subíntima fibrovascular o subsinovial, unida al tejido conectivo fibroso del LC. Estas membranas revisten por completo el LC de la ATM de un adulto, pero están ausentes en las superficies articulares y el disco.

La MS contiene una población heterogénea de células que gracias a la microscopía electrónica de transmisión se han podido identificar. Entre estas célula destacan los sinoviocitos ubicados en la capa íntima de la MS (Wurgaft). Estas células sinoviales aparecen dispuestas en una capa continua, aunque a menudo se hallan entremezcladas con fibras del tejido conectivo capsular y con células adiposas (Gómez de Ferraris, 2009). Debido a que estas células no limitan con una lámina basal, pueden no ser consideradas como una verdadera membrana (Nanci, 2008; Gómez de Ferraris). En la MS se forman vellosidades o pliegues que sobresalen hacia la cavidad articular, especialmente en su fórnix y su parte postero superior, aumentando el contacto entre el tejido conectivo sinovial y el LS. Estos pliegues aumentan en número con la edad y también son más prominentes en las articulaciones afectadas por procesos patológicos, con la consiguiente disminución en la producción de LS y reducción del nivel de lubricación de las superficies articulares (Nanci; Gómez de Ferraris). Estas modificaciones podrían ser una explicación a los cambios que se producen en la intensidad de los ruidos articulares durante del desarrollo de patologías articulares (Nanci; Gómez de Ferraris).

Composición de las membranas sinoviales. Esencialmente toda MS consta de una capa celular íntima sobre una capa subíntima vascular (Nanci). La capa sinovial subíntima está unida al tejido conectivo fibroso de la cápsula, es vascularizada, y presenta tejido conectivo con algunas células adiposas (Wurgaft; Gómez de Ferraris; Okeson, 2013). Es un tejido conectivo laxo que contiene elementos vasculares junto con fibroblastos dispersos, macrófagos, mastocitos, células grasas y algunas fibras elásticas que impiden el plegado de la membrana (Nanci). Se pueden encontrar diversas variedades de tejido conectivo, de acuerdo a esto la capa sinovial subíntima se clasifica en tipo areolar o laxa, tipo fibrosa, y tipo adiposa (variedad ausente en condiciones normales), aunque no se conoce la especificidad funcional de cada una (Nanci). La presencia de un tipo u otro, depende de las demandas a las resistencias mecánicas de la región, la edad, o de alguna patología (Gómez de Ferraris; Okeson). La capa subíntima está irrigada por una red de capilares que pueden ser de tres tipos: continuos, fenestrados y discontinuos. También se han observado vasos linfáticos originados en los recesos de los compartimentos supra e infradiscal, a corta distancia de la superficie sinovial (Gómez de Ferraris). Según este mismo autor, recientemente se ha identificado la presencia de células dendríticas en la capa subíntima, de función similar a las terminaciones libres mecanoreceptoras (Gómez de Ferraris).

Por su parte, la capa sinovial íntima limita con el espacio articular y varía en estructura, teniendo de una a cuatro capas de células entremezcladas en una matriz intercelular amorfa, libre de fibras (Wurgaft; Nanci). Dentro de su conformación se encuentran los sinoviocitos que se disponen en forma epiteloídea, carecen de medios de unión y no están apoyados en una lámina basal. Los sinoviocitos tipo A (semejantes a los macrófagos) son poco abundantes (20\%) y se originan de monocitos derivados de la médula ósea (Nanci; Gómez de Ferraris). Caracterizados por ondulaciones de la superficie, invaginaciones de la membrana plasmática y vesículas pinocitócicas. Además poseen un complejo golgiensi (aparato de Golgi) muy desarrollado y numerosas vesículas golgiensis lisosomales, que sintetizan y liberan enzimas líticas que fagocitan detritos celulares o células inflamatorias, sin embargo estas células tienen poco retículo endoplasmático rugoso. Las células tipo B o sinoviocitos tipo B son más abundantes $(70 \%)$, se asemejan a fibroblastos, no están conectadas por complejos de unión y tampoco descansan sobre una lámina basal, poseen un aparato cisternal más pequeño, un retículo endoplasmático rugoso muy desarrollado y abundantes gránulos. Poseen membrana plasmática menos ondulada, produciendo una secreción rica en glicoproteínas y glicosaminoglicanos, entre los que se destacan el ácido hialurónico (AH) y la lubricina. Por lo tanto, la cavidad articular no está revestida por epitelio, lo que le da la característica de semipermeable.

Formación y distribución del líquido sinovial. El LS ocupa una posición clave en la fisiología de las articulaciones. El LS se encuentra en las articulaciones sinoviales, bolsas y vainas tendinosas. El término sinovial fue introducido por Paracelso (1493 - 1541) y significa "como la clara de huevo", por ser claro y viscoso (Aghabeigi et al., 1993), proviene del latín synovia compuesto por el prefijo griego syn(con, junto) y la palabra latina ovum (huevo). Se define como una fina película de gel viscoso y filante de color amarillento claro (Kristensen et al.) desempeñando un papel impor- 
tante en el mantenimiento y regulación de la fisiología y función de las articulaciones (Liu et al., 2016). El LS es ligeramente alcalino en reposo y su $\mathrm{pH}$ baja durante la actividad articular. La composición química del LS indica que es un dializado del plasma suplementado con proteínas y proteoglicanos. La MS es responsable de controlar el paso de los componentes del plasma y de producir los componentes adicionales del LS (Nanci). Tiene funciones lubricantes, reguladoras y metabólicas disminuyendo el roce de las superficies articulares durante los movimientos (Gómez de Ferraris; Okeson; Kristensen et al.). Normalmente, el LS se deposita en los márgenes de la ATM y lubrica la articulación mediante dos mecanismos distintos: lubricación límite y lubricación lágrima. La lubricación límite se produce durante los movimientos articulares, donde el LS es impulsado desde los recesos de los espacios supra e infradiscal, hacia la superficie articular (Kristensen et al.). En cambio, la lubricación lágrima o exudativa se produce gracias a la capacidad de las superficies articulares de absorber una pequeña cantidad de LS secundario a las fuerzas producidas entre las superficies articulares, favoreciendo el intercambio metabólico. En reposo los sinoviocitos tipo B también elaboran pequeñas gotas de LS para favorecer aún más la lubricación, pero fuerzas de compresión prolongadas sobre las superficies articulares agotan su producción favoreciendo su adhesión (Okeson).

Volumen y composición del líquido sinovial en la ATM. En la ATM el LS presenta un volumen virtual aproximado de 1 $\mathrm{mL}$, por lo que la aspiración directa reúne muy poco o ningún fluido, siendo necesario la dilución en solución salina para su estudio. En casos de derrame preexistente secundario a alguna enfermedad sistémica como la artritis reumatoidea este volumen puede estar aumentado (Nanci). Aghabeigi et al. (2002) reportaron un volumen medio de LS en la ATM en el aspirado salino de $32 \mu \mathrm{L}$, sin diferencia significativa entre las articulaciones sintomática y asintomática.

El LS esta compuesto por proteínas plasmáticas, azúcares, lípidos, componentes celulares, moléculas lubricantes, citoquinas, factores de crecimiento y enzimas proteolíticas, las cuales se resumen en la Tabla I. Recientemente se han identificado también células madre mesenquimales en aspirados de LS, provenientes tanto de la capa íntima como subíntima, otorgando al LS un potencial proliferativo y condrogénico (Yao et al., 2018).

Las principales proteínas presentes son la albúmina y globulinas tipo b1, g, a1 y a2 (Hui et al.), siendo su concentración un tercio de las encontradas en el plasma sanguíneo. Proteínas de alto peso molecular como el fibrinógeno, se encuentran en menor cantidad, a diferencia de la albúmina y la transferrina que son proteínas de bajo peso molecular las cuales se encuentran en mayor concentración (Hui et al.). La concentración depende del tamaño de la proteína, del flujo sanguíneo, de la concentración plasmática, de la permeabilidad vascular, del drenaje linfático, de su producción y degradación local (Gómez de Ferraris; Okeson; Kristensen et al.). Otro componente importante del LS son las moléculas lubricantes, sintetizadas y secretadas por los sinoviocitos tipo B. Estas corresponden principalmente al AH y proteoglicano 4 (PRG4) (Hui et al.). El AH es considerado un determinante importante de las propiedades viscoelásticas y tixotrópicas dependientes de la tasa de flujo del LS. El AH es un mucopolisacárido de alto peso molecular compuesto por una cadena disacarídica larga (ácido glucurónico y $\mathrm{N}$-acetilglucosamina en unidades repetidas), con una semivida intraarticular aproximada de 20 horas. Se sintetiza por los fibroblastos de la capa íntima, alcanzando concentraciones muy elevadas dentro de la ATM y en la matriz extracelular de la capa íntima. Se degrada localmente en la MS, órganos linfoides e hígado. Forma una red intrincada, con mallas que dejan pasar fácilmente solutos de bajo peso molecular, pero retienen las moléculas de alto peso molecular. Ejerce un fuerte poder osmótico y forma un gel deformable que se vuelve elástico cuando aumentan las fuerzas de cizallamiento, contribuyendo a la viscosidad del líquido (Ea et al., 2008; Hui et al.). Se ha propuesto que el AH también podría promover la proliferación celular e inhibir la apoptosis inducida por óxido nítrico de los condrocitos articulares (Guo et al., 2015). Por otro lado, PRG4 es un lubricante eficaz que protege la superficie del cartílago articular

Tabla I. Componentes del líquido sinovial.

\begin{tabular}{|c|c|}
\hline Componentes del LS & Tipos \\
\hline Proteínas plasmáticas & Albúmina; globulinas tipo $\beta 1, \gamma, \alpha 1$ y $\alpha 2$; fibrinógeno, transfe rina. \\
\hline Azúcares y lípidos & Glucosa, galactosa, fructosa, lípidos. \\
\hline Células & Células libres descamadas, macrófagos, monocitos, linfocitos, leucocitos polimorfonucleares. \\
\hline Moléculas lubricantes & AH, PRG4, SZP, lubricina, mucinas. \\
\hline Citoquinas y factores de crecimiento & $\begin{array}{l}\text { Citoquínas proinflamatorias: IL-1 } \alpha \text {, IL-1 } \beta \text {, IL-6, TNF- } \alpha \text {; citoquinas antiinflamatorias: IL-2 } \\
\text { IL-4, IL-10, IL-13; TGF- } \alpha 1 \text {; IFN-c. }\end{array}$ \\
\hline Enzimas proteolíticas & MMP-1, MMP-3, serina, cisteína, plasmina, calicreína, catepsina B, TIMP. \\
\hline
\end{tabular}


sinérgicamente. Productos del PRG4, como la proteína de la zona superficial (SZP) y la lubricina, son glucoproteínas mucinosas que median la lubricación límite de las articulaciones (Ea et al.). La SZP es una glucoproteína sintetizada y secretada por los condrocitos en la superficie del cartílago, a su vez, lubricina es una glucoproteína expresada por fibroblastos sinoviales la cual actúa como elemento protector de los procesos degenerativos de las superficies articulares (Ea et al.). La lubricina es un componente muy importante del LS y esencial en el mantenimiento de la ATM protegiendo las superficies del cartílago articular y regulando el crecimiento de las células sinoviales. Además del papel en la lubricación, PRG4 regula la proliferación y adhesión de células sinoviales (Hill et al., 2014).

Citoquinas y factores de crecimiento también están presentes en el LS, siendo factores reguladores importantes para los sistemas inmunológico y metabólico en la articulación, regulando algunas poblaciones celulares como condrocitos y células sinoviales (Ea et al.). Las citoquinas son secretadas por células inmunocompetentes de la MS, por condrocitos o proceden del plasma. Kristensen et al. detectaron en muestras de LS de ATM sanas que los niveles de IL-10, IL-1b e IL-6 fueron en su mayoría indetectables; TNF- $\alpha$ es constante; IFN-Gamma y IL- 2 se detectaron esporádicamente; además los niveles de TNF- $\alpha$ e IFN-c se encuentran directamente relacionados, lo que sugiere una homeostasis entre las dos citocinas.

Las enzimas proteolíticas que degradan la matriz, como las metaloproteinasas de matriz (MMP)-1 y MMP-3, se secretan principalmente a partir de condrocitos como zimógenos o proenzimas latentes con dominios propeptídicos que se escinden durante la activación extracelular (Hui et $a l$.$) . Otras proteinasas, tales como serina y cisteína están$ implicadas en la activación de proMMPs. Por ejemplo, la plasmina, la calicreína y la catepsina B activan proMMP-1. También están presentes inhibidores de tejidos de metaloproteinasas (TIMP) e inhibidores de proteinasas que activan proMMPs (Hui et al.). Las MMPs están funcionalmente equilibradas con sus inhibidores tisulares (TIMP) durante el metabolismo normal. Esto conduce a la homeostasis de la matriz extracelular y el reemplazo de células viejas o dañadas (Ferreira et al., 2016). Estas enzimas juegan un papel importante tanto en condiciones fisiológicas como patológicas en la ATM (Ferreira et al.).

Fisiopatología del líquido sinovial de la ATM. El LS está en contacto físico directo con los cartílagos de las superficies articulares y las MS, mediando las interacciones entre los tejidos de las articulaciones sinoviales (Hui et al.). La composición y función del LS se ve alterado durante una lesión articular o durante un proceso patológico instaurado, ya sea por cambios directos o cambios en los tejidos de la ATM. Estos cambios pueden resultar en una capacidad reducida de lubricar las superficies articulares y generar un ambiente catabólico dentro de la articulación, contribuyendo conjuntamente al deterioro de las articulaciones (Hui et $a l$.). Se ha identificado que los niveles de mediadores inflamatorios en LS se correlacionan con la presencia de dolor, cronicidad, gravedad del cambio degenerativo y respuesta al tratamiento (Bouloux, 2009; Güven et al., 2015). Estudios han demostrado que las concentraciones de citoquinas derivadas de monocitos y macrófagos se elevan en el LS de ATM enfermas. IL-1 $\beta$, IL-6 y TNF- $\alpha$ pueden promover la liberación de proteinasas estimulando la expresión de enzimas degradantes y mediadores inflamatorios, lo que resulta en la inflamación de la ATM y degradación de huesos y cartílagos (Kellesarian et al., 2016). Citoquinas proinflamatorias como el TNF- $\alpha$ aumentarían significativamente en osteoartritis de ATM y estarían relacionadas con la etapa de severidad de la enfermedad y el nivel de dolor (Güven et al.). IL-1 $\beta$, IL-6, IL-8 y TNF- $\alpha$ también se han medido en pacientes sintomáticos con luxaciones discales con reducción, luxaciones discales sin reducción y osteartritis. Los niveles de las 4 citoquinas fueron significativamente mayores que en los controles asintomáticos (Bouloux). Curiosamente, la presencia o ausencia de reducción discal no influyó en los niveles de citoquinas, aunque la osteoatritis se asoció con un aumento del nivel de IL-6. Según este mismo autor, una posible explicación del aumento de los niveles de IL-6 en la osteatritis sería su carácter inflamatorio destructivo crónico, siendo la IL-6 una potente citoquina anti-inflamatoria, por lo que puede representar un intento frustrado de modular la progresión de la enfermedad. IL-6 también se ha demostrado que se correlaciona con el grado de sinovitis y cronicidad de los síntomas (Güven et al.). Sin embargo, muchas veces la gravedad de las patologías observadas artroscópicamente y los niveles de cada citoquina investigada no parecen reflejarse en el estado clínico (Güven et al.).

Guo et al. indicaron que las citoquinas proinflamatorias TNF- $\alpha$, IL-1 $\beta$ y IL- 1 a suprimen los niveles de PRG4 secretados en cultivos de condrocitos. Bradicinina, leucotrieno B4, prostaglandina E2 y sustancia P también se han identificado en el LS de pacientes con alteración internas y osteoartritis de ATM (Bouloux).

Algunos mediadores específicos del dolor, tales como el neuropéptido Y, serotonina, péptido relacionado con el gen de la calcitonina (CGRP) y el receptor de potencial transitorio vanilloid-1 (TRPV1) también se han identificado en ATM sintomáticas y se correlacionan con el nivel de dolor o grado de sinovitis (Bouloux). El ligando de receptor activador para el factor nuclear $\mathrm{k} B$ (RANKL) es un marca- 
dor sustituto de la actividad osteoclástica y se ha medido en el LS de pacientes con alteraciones internas y osteoartritis de ATM sometidos a artroscopia. La expresión de este marcador se observó en sinoviocitos, células endoteliales y fibroblastos, aunque no hubo correlación con el grado artroscópico de sinovitis u osteoartritis (Bouloux).

La sobrecarga por compresión de los sinoviocitos de la ATM parece modular la producción de citoquinas inflamatorias como MMP. Estas proteinasas hidrolizan componentes de la matriz extracelular, pudiendo infiltrarse en la sinovial y alterar la viscosidad del LS, provocando alteraciones en la lubricación y nutrición del cartílago y disco articular (Ferreira et al.).

PRG4 en osteoartritis de ATM. En procesos degenerativos articulares se ha observado que PRG4 presenta una degradación y síntesis reducida, debido a la pérdida de sinoviocitos y citoquinas inflamatorias. Guo et al. observaron que la expresión de PRG4 en sinoviocitos de ATM osteoartrítica fue suprimida dramáticamente por la hipoxia, que puede estar relacionada con diversas citoquinas inflamatorias segregadas en la ausencia de oxígeno. El bloqueo del gen PRG4 en ratones PRG4 -/- produjo una degeneración de la ATM similar a una osteoartritis que aumentó en gravedad con el tiempo, siendo más severa en la ATM que en la articulación de la rodilla, por lo que se relaciona a esta molécula con la regulación el crecimiento sinovial (Hill et al.). Asi mismo, se ha demostrado que la suplementación intraarticular con PRG4 redujo el daño en el cartílago articular según mediciones histológicas y radiográficas (Guo et al.).

Acido hialurónico en osteoartritis de ATM. La presencia de AH es vital en la fisiología de la ATM poduciendo un aumento en la proliferación de sinoviocitos en normoxia o hipoxia; regulando negativamente la expresión génica de TNF- $\alpha$ e IL-8 en sinoviocitos; e inhibiendo la producción de MMP-1, MMP-3 y MMP-13 estimulada por IL- $1 \beta$ en el cartílago articular. En presencia de cambios degenerativos se produciría una disminución en el peso molecular y descomposición del AH con una alteración en su distribución (Hui et al.), decreciendo la viscosidad del LS y provocando un debilitamiento de la protección de la matriz de proteoglicanos articulares (Iturriaga et al., 2017a). El uso de AH exógeno como terapia podría mejorar la secreción de AH por parte de los sinoviocitos y mediar la nutrición a la superficie avascular de la articulación (Iturriaga et al., 2017b). A pesar que el AH exógeno se degrada rápidamente por la hialuronidasa en 12-24 horas una vez que se inyecta en el espacio articular, se ha evaluado un efecto positivo de aproximadamente 6 meses (Guo et al.). Se ha observado que el AH también podría sobrerregular la expresión de PRG4 en sinoviocitos de ATM osteoartríticas dentro de las
$24 \mathrm{~h}$ in vitro, lo que sugiere que el efecto lubricante del $\mathrm{AH}$ exógeno podría estar asociado con la regulación positiva de PRG4 (Guo et al.). Esta expresión se puede ver afectada por las condiciones de oxígeno de los estudios. Aunque la expresión del gen PRG4 fue regulado por el AH, la secreción de PRG4 en condiciones de normoxia fue suprimida. La razón podría estar relacionada con la compleja regulación de la secreción de PRG4, siendo determinada por una combinación de estímulos mecánicos y químicos (Guo et al.).

Presencia de bacterias en el líquido sinovial. Las bacterias están presentes en el LS con alta frecuencia y podrían estar asociadas con algunos síntomas clínicos (Kim et al., 2003). La invasión de bacterias en el espacio articular puede ocurrir a través de uno de varios mecanismos. La propagación hematógena de las bacterias es un mecanismo altamente probable como vía de infección. La sinovial ricamente vascularizada de la articulación no tiene una membrana basal limitante, lo que la hace vulnerable a la propagación hematógena (Kim et al.; Iturriaga et al., 2012). También se informa la propagación directa desde un sitio contiguo de infección, tal como la otitis externa o artritis infecciosa de la ATM. Si las bacterias entran en la cavidad articular, deben escapar de la respuesta inmune del hospedador para mantenerse vivas (Kim et al.; Iturriaga et al., 2012). Dentro de los cuadros que se pueden asociar a la presencia de bacterias dentro del LS encontramos a la artritis infecciosa de la ATM; situación clínica pocas veces descrito en la literatura (Iturriaga et al., 2012). Sin embargo, la presencia de bacterias en la articulación no necesariamente causa una artritis infecciosa, deben existir una serie de otros factores para que ésta se lleve a cabo, como lo son enfermedades graves concomitantes, cirrosis, fallas renales, diabetes, entre otras.

\section{CONCLUSIONES}

Conocer la histología y fisiología de la MS, y la producción y composición del LS nos permite comprender los procesos que ocurren durante las distintas patologías que puede experimentar la ATM. Niveles variados de citoquinas, enzimas, moléculas lubricantes en el LS se han presentado en la literatura, aunque sigue siendo tema de discusión. Las razones para esto pueden incluir un bajo número de pacientes en los estudios y variaciones en las técnicas implementadas. Asi mismo, las técnicas de muestreo potencialmente inadecuadas para el análisis del LS de la ATM y las limitaciones con la sensibilidad de los ensayos utilizados para detectar sus componentes dificultan la interpretación de los resultados. En relación a los cambios en la composición del LS durante un proceso inflamatorio podemos destacar que la ATM es una articulación sinovial especial 
tanto en términos de su estructura, como en las vías genéticas del desarrollo que rigen su formación, por lo que es necesario continuar su estudio y comprensión.

AGRADECIMIENTOS. Queremos agradecer a CONICYT a través de la Beca PFCHA/ Doctorado Nacional/201521151352 por el apoyo en esta investigación.

ITURRIAGA, V.; MENA, P.; OLIVEROS, R.; CERDA, C.; TORRES, D. \& DEL SOL, M. Value of synovial fluid in the temporomandibular joint and its implications in articular pathology. Int. J. Morphol., 36(1):297-302, 2018.

SUMMARY The aim of this study was to conduct a review of the literature on the value of synovial fluid in the temporomandibular joint and its implications in joint pathology. The synovial fluid is a viscous and filamentous fluid of a light yellow color, composed mainly of plasma proteins, cellular components, lubricating molecules, cytokines, growth factors and enzymes. It is produced by the synovial membrane and is in direct relation with the joint surfaces and the synovial membrane, mediating the interactions between the tissues of the synovial joints. In the temporomandibular joint it plays a fundamental role in the maintenance and regulation of physiology, through lubricating, regulating and metabolic functions, decreasing the friction of the joint surfaces during all its movements. In temporomandibular disorders, synovial fluid function can be altered by changes in the tissues of the temporomandibular joint, these changes can result in a decrease in the ability to lubricate the joint surfaces and generate a catabolic environment inside the joint, contributing together to the deterioration of these.

KEY WORDS: Synovial fluid; Temporomandibular joint; Temporomandibular disorders; Osteoarthritis.

\section{REFERENCIAS BIBLIOGRÁFICAS}

Aghabeigi, B.; Cintra, N.; Meghji, S.; Evans, A. \& Crean, S. J. Temporomandibular joint synovial fluid sampling: estimation of dilution factor using calcium ion concentration. Int. J. Oral Maxillofac. Surg., 31(6):646-9, 2002.

Aghabeigi, B.; Henderson, B.; Hopper, C. \& Harris, M. Temporomandibular joint synovial fluid analysis. Br. J. Oral Maxillofac. Surg., 31(1):15-20, 1993.

Bouloux, G. F. Temporomandibular joint pain and synovial fluid analysis: a review of the literature. J. Oral Maxillofac. Surg., 67(11):2497-504, 2009.

Ea, H. K.; Bazille, C. \& Lioté, F. Histologie et physiologie de la membrane synoviale. EMC (Elsevier Masson SAS, Paris), Appareil locomoteur, 14004-A-10, 2008.

Ferreira, L. M.; Moura, Á. F.; Barbosa, G. A.; Pereira, H. S. \& Dos Santos Calderón, P. Do matrix metalloproteinases play a role in degenerative disease of temporomandibular joint? A systematic review. Cranio, 34(2):112-7, 2016

Gómez de Ferraris, M. Histología y Embriología Bucodental. $2^{\mathrm{a}}$ ed. Santiago de Chile, Médica Panamericana, 2009.
Guo, H.; Fang, W.; Li, Y.; Ke, J.; Deng, M.; Meng, Q.; Li, J. \& Long, X. Upregulation of proteoglycan 4 in temporomandibular osteoarthritic synovial cells by hyaluronic acid. J. Oral Pathol. Med., 44(8):622-7, 2015.

Güven, O.; Tekin, U.; Salmanoglu, B. \& Kaymak, E. Tumor necrosis factoralpha levels in the synovial fluid of patients with temporomandibular joint internal derangement. J. Craniomaxillofac. Surg., 43(1):102-5, 2015.

Hill, A.; Duran, J. \& Purcell, P. Lubricin protects the temporomandibular joint surfaces from degeneration. PLoS One, 9(9):e106497, 2014.

Hui, A. Y.; McCarty, W. J.; Masuda, K.; Firestein, G. S. \& Sah, R. L. A systems biology approach to synovial joint lubrication in health, injury, and disease. Wiley Interdiscip. Rev. Syst. Biol. Med., 4(1):15-37, 2012.

Iturriaga, V.; Bornhardt, T.; Manterola, C. \& Brebi, P. Effect of hyaluronic acid on the regulation of inflammatory mediators in osteoarthritis of the temporomandibular joint: a systematic review. Int. J. Oral Maxillofac. Surg., 46(5):590-5, $2017 \mathrm{a}$.

Iturriaga, V.; Vásquez, B.; Manterola, C. \& del Sol, M. Role of hyaluronic acid in the homeostasis and therapeutics of temporomandibular joint osteoarthritis. Int. J. Morphol., 35(3):870-6, 2017b.

Iturriaga, W. V.; Bornhardt, S. T.; Fuentes, N. J.; Fuentes, F. R. \& Raposo, C. A. Artritis infecciosa y su relación con la articulación temporomandibular. Av. Odontoestomatol., 28(1):25-32, 2012.

Kellesarian, S. V.; Al-Kheraif, A. A.; Vohra, F.; Ghanem, A.; Malmstrom, H.; Romanos, G. E. \& Javed, F. Cytokine profile in the synovial fluid of patients with temporomandibular joint disorders: A systematic review. Cytokine, 77:98-106, 2016.

Kim, S. J.; Park, Y. H.; Hong, S. P.; Cho, B. O.; Park, J. W. \& Kim, S. G. The presence of bacteria in the synovial fluid of the temporomandibular joint and clinical significance: preliminary study. J. Oral Maxillofac. Surg., 61(10):1156-61, 2003.

Kristensen, K. D.; Alstergren, P.; Stoustrup, P.; Küseler, A.; Herlin, T. \& Pedersen, T. K. Cytokines in healthy temporomandibular joint synovial fluid. J. Oral Rehabil., 41(4):250-6, 2014.

Liu, W.; Sun, Y.; He, Y.; Zhang, H.; Zheng, Y.; Yao, Y. \& Zhang, Z. IL-1b impedes the chondrogenic differentiation of synovial fluid mesenchymal stem cells in the human temporomandibular joint. Int. J. Mol. Med., 39(2):317-26, 2016.

Nanci, A. Ten Cate's Oral Histology Development, Structure and Function. $8^{\mathrm{a}}$ ed. Quebec, Elsevier; 2008.

Okeson, J. P. Tratamiento de Oclusión y Afecciones Temporomandibulares. $7^{\mathrm{a}}$ ed. Barcelona, Elsevier, 2013.

Wurgaft, R. Desarrollo y Estructura de la Articulación Temporomandibular. $3^{\mathrm{a}}$ ed. Santiago de Chile, Servimpres, 2003.

Yao, Y.; Li, Z. Y.; Zhang, H.; Zheng, Y. H.; Mai, L. X.; Liu, W. J.; Zhang, Z. G. \& Sun, Y. P. Synovial fluid-derived synovial fragments represent an improved source of synovial mesenchymal stem cells in the temporomandibular joint. Int. J. Mol. Med., 41(1):173-83, 2018.

\section{Dirección para Correspondencia}

Prof. Dr. Mariano del Sol C.

Centro de Excelencia en

Estudios Morfológicos y Quirúrgicos (CEMyQ)

Universidad de La Frontera

Temuco

CHILE

Email: mariano.delsol@ufrontera.cl

Recibido : 05-10-2017

Aceptado: 27-12-2017 